\title{
Dieta del quique (Galictis cuja Molina 1782) en un remanente de bosque valdiviano fragmentado del sur de Chile
}

\section{Diet of the lesser grison (Galictis cuja Molina 1782) in a Valdivian fragmented forest remnant in southern Chile}

\author{
Soraya Sade ${ }^{1}$, Jaime R. Rau², José I. Orellana ${ }^{3}$ \\ ${ }^{1}$ Programa de Magíster en Ciencias, Vicerrectoría de Investigación y Postgrado, Escuela de Postgrado, Universidad de Los \\ Lagos, Edificio "José Manuel", Cochrane 1.070, Osorno, Chile. 'Laboratorio de Ecología, Departamento de Ciencias Básicas \\ \& Programa IBAM, Universidad de Los Lagos, Casilla 933, Osorno, Chile. *E-mail: jrau@ulagos.cl. ${ }^{3}$ Laboratorio de Vida \\ Silvestre, Departamento de Ciencias Básicas, Universidad de Los Lagos, Casilla 933, Osorno, Chile.
}

\begin{abstract}
RESUMEN
Pese a su amplia distribución y uso de diferentes hábitats en América del Sur existe poca información sobre el quique, Galictis cuja (Carnivora: Mustelidae). Inicialmente descrito como un mamífero generalista que vive cerca de ríos y con una dieta católica, estudios en Uruguay, Chile central y Argentina han mostrado que se especializa en roedores nativos y lagomorfos introducidos. En este trabajo se documenta por primera vez su dieta otoño-invernal en un remanente de bosque valdiviano fragmentado. En estas estaciones climáticas, donde la abundancia de roedores aumenta, la mayor frecuencia estandarizada de presas en la dieta del quique (82\%) correspondió a 6 especies de roedores nativos cuyas masas corporales variaron entre 24 y 58 gramos. Pese a haber liebres europeas introducidas (Lepus europaeus) en la matriz agrícola donde se encuentra el fragmento estudiado, no se encontraron restos de ellas en las 45 muestras fecales analizadas, sugiriendo que los quiques cazaron sus presas al interior y/o en el borde del fragmento.
\end{abstract}

Palabras Claves: Carnívoros, ecorregión valdiviana, fragmentación, hábitos alimenticios.

\begin{abstract}
Despite its wide distribution and use of different habitats in South America, there is little information on the lesser grison Galictis cuja (Carnivora: Mustelidae). Initially described as a generalist mammal that lives near rivers and with a catholic diet, studies in Uruguay, central Chile and Argentina have shown that this mustelid preys on native rodents and introduced lagomorphs. In this paper, the fall and winter diet of the lesser grison in a fragmented Valdivian forest remnant is documented for the first time. In these seasons, when the abundance of rodents increases, the highest standard frequency of prey in the diet of this carnivore mammal $(82 \%)$ corresponds to 6 species of native rodents whose body masses ranged between 24 and 58 grams. Despite the presence of introduced European hares (Lepus europaeus) in the agricultural matrix, remains of hares were not found in the 45 fecal samples analyzed, suggesting that lesser grisons hunted their prey inside and/or at the edge of the fragment.
\end{abstract}

KEYwords: Carnivores, food habits, fragmentation, Valdivian rainforest.

\section{INTRODUCCIÓN}

El quique, Galictis cuja (Carnivora: Mustelidae), es un pequeño mamífero carnívoro endémico de Sudamérica que se encuentra distribuido ampliamente en distintos hábitats del continente (Yensen \& Tarifa 2003). En Chile su estado de conservación corresponde a una especie Inadecuadamente Conocida para la I, II y III regiones y Vulnerable para el resto del territorio (Reglamento de la Ley de Caza Decreto
Supremo $\mathrm{N}^{\circ} 05$ de 1998). A pesar de su amplia distribución geográfica es, sin embargo, una especie poco conocida. Respecto a su dieta se han publicado los siguientes trabajos: (i) uno en humedales del Este de Rocha, Uruguay (Kraus \& Rödel 2004), (ii) otro en Chile central (Ebensperger et al. 1991) y (iii) tres correspondientes a diferentes localidades de la Patagonia Argentina (Delibes et al. 2003, Diuck-Wasser \& Cassini, 1998, Zapata et al. 2005). En general todos estos trabajos coinciden en que el principal componente de 
la dieta del quique corresponde a mamíferos (más del 90\%), siendo sus principales presas roedores nativos y lagomorfos introducidos (2 especies), y un porcentaje casi despreciable correspondería a crustáceos, anfibios, reptiles y aves.

El objetivo de este trabajo fue documentar por primera vez para bosques templados del sur de América del Sur la dieta del quique durante las estaciones de otoño e invierno.

\section{MATERIALES Y MÉTODOS}

El área de estudio está situada en la depresión intermedia de la provincia de Osorno, sur de Chile (40 46' 14"'$72^{\circ} 57^{\prime} 21^{\prime}$ '), correspondiente a la ecorregión del bosque valdiviano. Durante otoño (abril-mayo, $\mathrm{n}=15$ ) e invierno (junio-septiembre, $\mathrm{n}=30$ ) de 2011 se colectaron 45 fecas de quiques en un fragmento (ca. 31 hectáreas) de bosque nativo valdiviano cercano a la carretera que comunica las ciudades de Osorno y Puerto Octay. Las mismas se acumularon en una letrina (Diuck-Wasser \& Cassini 1998, Delibes et al. 2003, Zapata et al. 2005) establecida sobre un tronco caído de pitra, Myrcengenia exsucca (DC.) O. Berg, árbol de la familia de las Mirtáceas y que es propio de los bosques de galería del sur de Chile. Estos bosques se caracterizan por presentar marcadas inundaciones estacionales (Fig. 1.). Las fecas colectadas en la letrina (Fig. 2) representan diferentes períodos de tiempo (abril-septiembre) correspondiendo a deposiciones de diferentes tamaños. Para una submuestra aleatoria de $\mathrm{n}=16$ fecas sus dimensiones (media aritmética y un error estándar) fueron: longitud 69,3 $\pm 5,2$ (coeficiente de variación, $\mathrm{CV}=30,0 \%$; rango $35,9-100,3 \mathrm{~mm}$ ); ancho $9,9 \pm 0,5(\mathrm{CV}=18,2 \%$; rango 7,2-12,8 $\mathrm{mm})$. Considerando la variación de sus longitudes ( $>\mathrm{CV}$ ) existirían al menos 4 clases de distribuciones de frecuencia de tamaños de fecas (Fig.3.), infiriéndose que éstas corresponderían a distintos individuos. La acumulación mensual de fecas varió entre 13-17, valor coincidente con los resultados obtenidos por Delibes et al. (2003) quienes encontraron letrinas con 15-20 fecas y Zapata et al. (2005) con 6-15 fecas.

En la carretera equidistante a $10 \mathrm{~m}$. de la letrina se encontró un ejemplar de quique atropellado (cf. juvenil) del cual se extrajo material fecal para compararlo con las fecas acumuladas en la letrina (Fig. 4), también se identificaron las presas del contenido estomacal.

Se utilizaron claves ad hoc (e.g., Reise 1973) para identificar los contenidos (principalmente roedores nativos) presentes en las 45 muestras fecales colectadas. Puesto que los cráneos y filas de molares estuvieron en su mayoría rotos, para la cuantificación de la dieta se utilizó la frecuencia de ocurrencia de presas en las muestras fecales (la que puede sumar más de un 100\% si en una muestra hay más de un tipo de presa) y, siguiendo a Maehr $\&$ Brady (1986), se la estandarizó (sumatoria $=100 \%$ ) dividiendo a las ocurrencias individuales de presas por su sumatoria total.
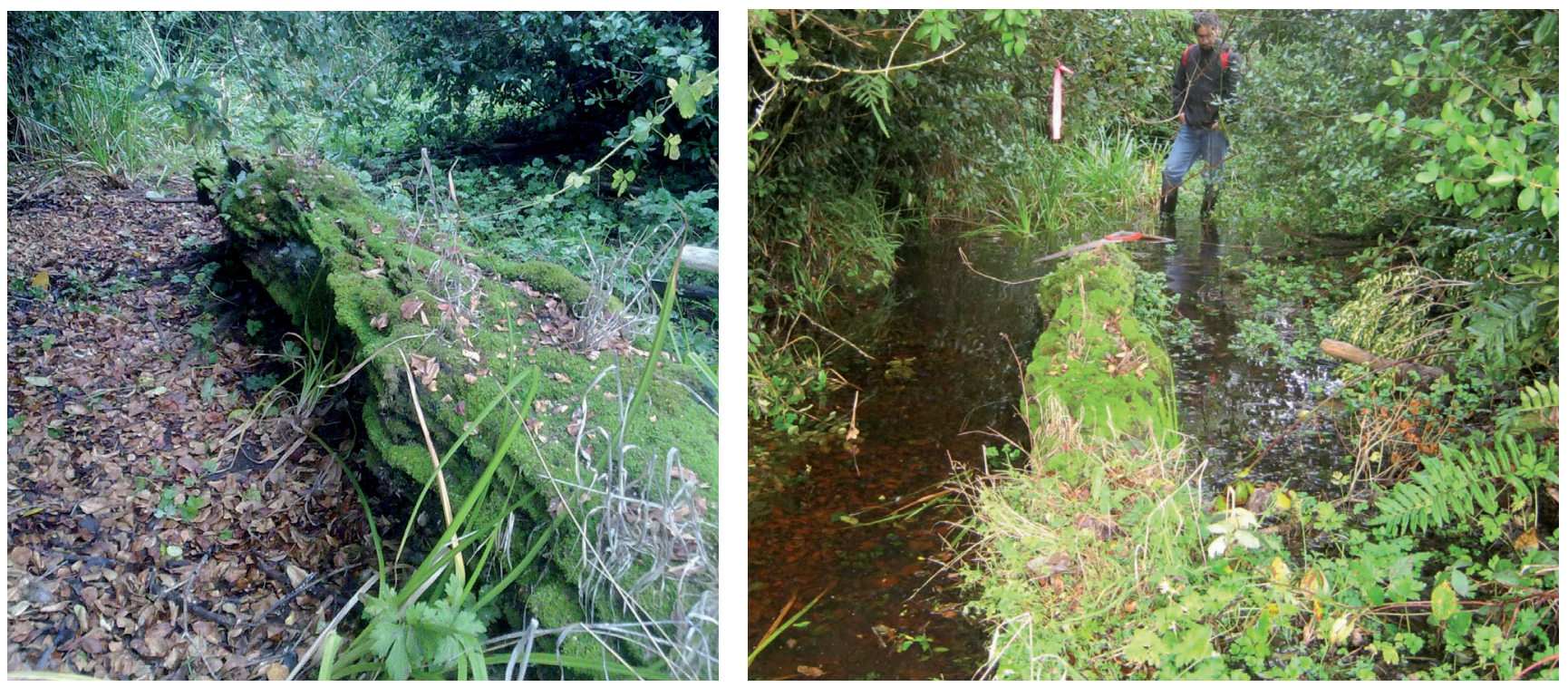

FIGURA 1. Letrina de quiques (Galictis cuja) en época estival izq., y estaciones otoño-invierno, der.

FIGURE 1. Latrine of lesser grisons (Galictis cuja) at summer, left and fall-winter, right. 


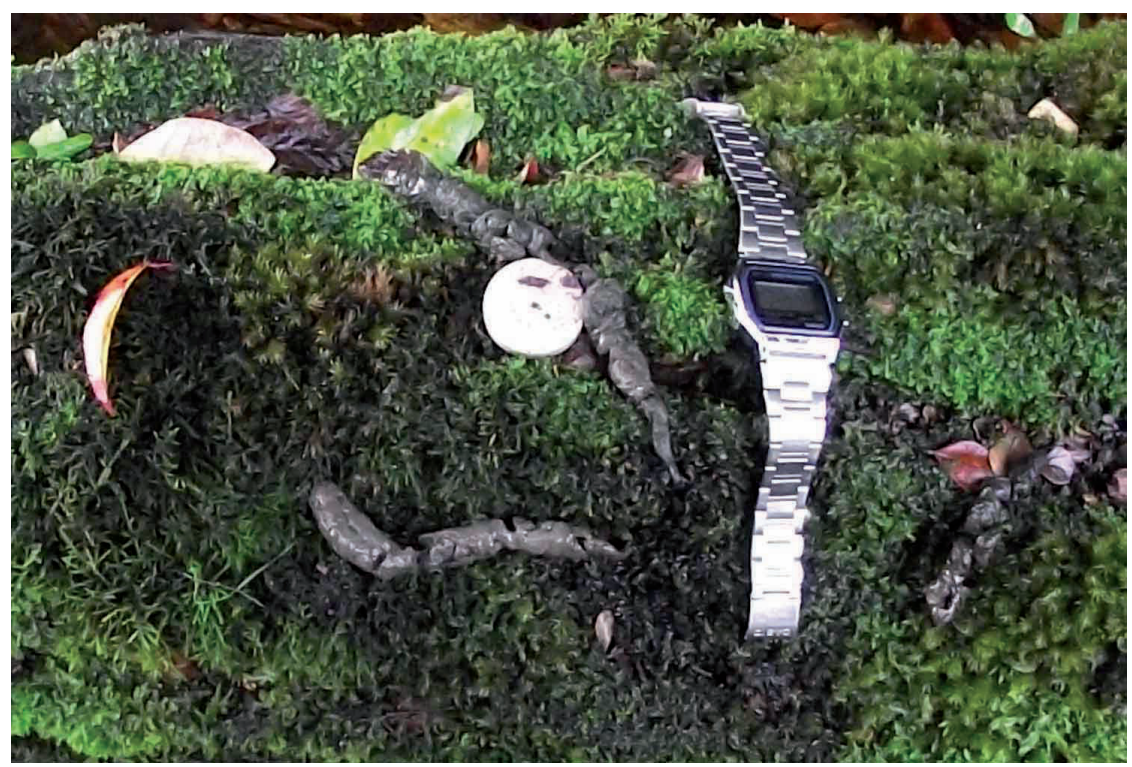

FiguRA 2. Fecas de quiques (Galictis cuja) presentes en su letrina situada al interior de un remanente de bosque valdiviano fragmentado del sur de Chile.

FIGURE 2. Scats of lesser grisons (Galictis cuja) at its latrine inside a fragmented Valdivian forest remnant.

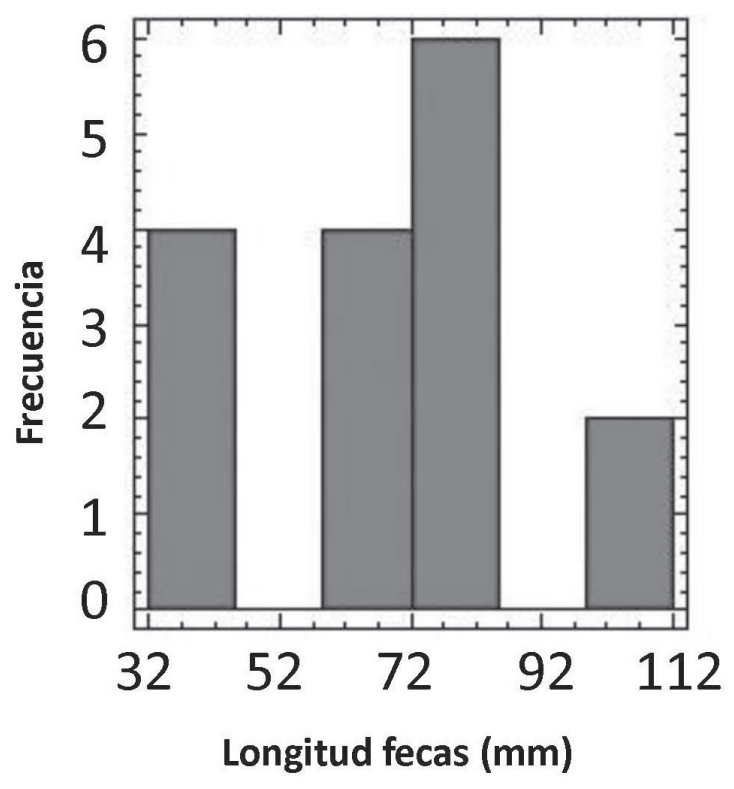

FIGURA 3. Histograma de distribución de frecuencias de las longitudes de fecas de Galictis cuja $(\mathrm{n}=16)$.

FIGURE 3. Frequencies distribution histogram of the lengths of the Galictis cuja $(\mathrm{n}=16)$.

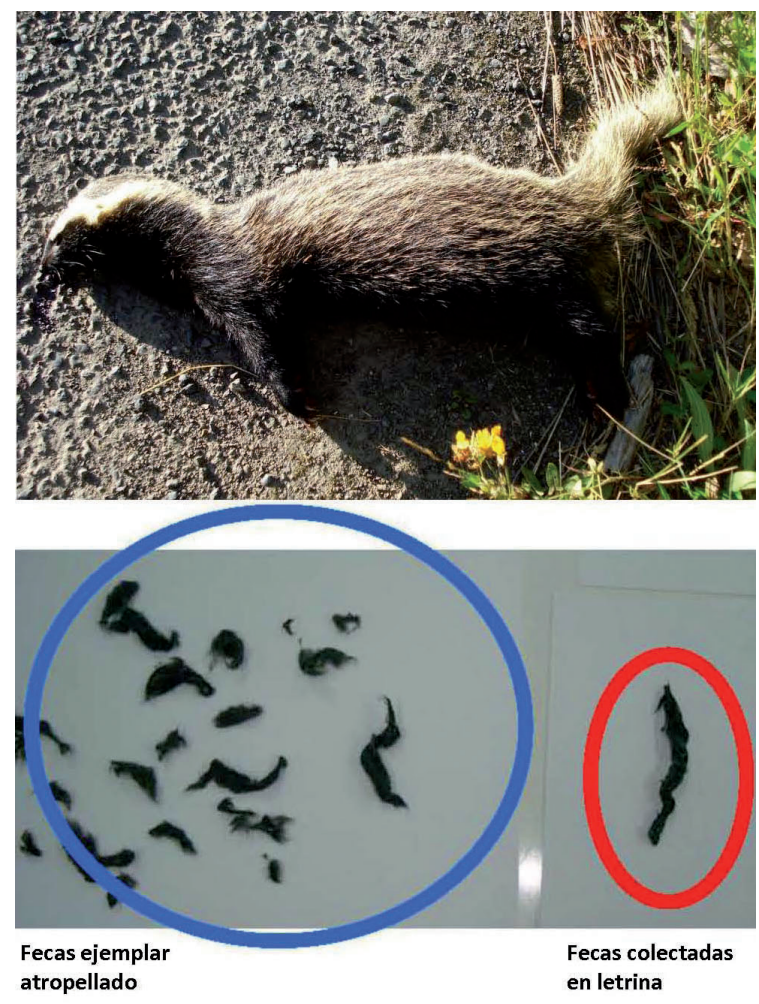

FIgURA 4. Ejemplar atropellado de quique (Galictis cuja) y su contenido fecal comparado con feca encontrada en letrina.

Figure 4. A lesser grison (Galictis cuja) after a road casualty and its faecal content compared with a scat collected at the latrine. 
Dieta del Quique en Chile: Soraya SADE ET AL.

\section{RESULTADOS}

En la ecorregión del bosque valdiviano la dieta otoño invernal del quique se caracteriza por el consumo de roedores sigmodontinos nativos (Tabla 1). Entre éstos se destaca Abrothrix olivaceus Waterhouse 1835 (masa corporal = 24 gramos), seguido por A. longipilis Waterhouse 1837 (35 gramos) y Loxodontomys micropus Waterhouse 1837 (58 gramos), probablemente consumidos en respuesta a su característico aumento otoño-invernal en el bosque templado húmedo del sur de Chile (cf. Murúa \& González 1986). En relación con ello, en el noroeste de la Patagonia argentina, Diuck-Wasser \& Cassini (1998) encontraron un mayor consumo de conejos (Oryctolagus cuniculus Linnaeus 1758), también cuando las abundancias de estas presas fueron más altas. Es importante destacar que en el área de estudio no están presentes los conejos, importantes en la alimentación del quique en Chile central (Ebensperger et al. 1991) y el noroeste de la Patagonia argentina (Diuck-Wasser \& Cassini 1998). Sin embargo, sí está presente (Dietrich 1984) la liebre europea (Lepus europaeus Pallas 1778), lagomorfo introducido y relevante en términos de la biomasa consumida por los quiques en el sureste de la Patagonia argentina (Zapata et al. 2005). Sin embargo, esta presa no estuvo presente en la dieta del quique en nuestro estudio. La liebre utiliza espacios abiertos, como la matriz de uso agrícola donde se encuentra el fragmento de bosque valdiviano aquí estudiado, lo que sugiere que los quiques obtuvieron sus presas del interior $\mathrm{y} / \mathrm{o}$ el borde de este remanente.

La presencia de insectos en la dieta de los quiques puede corresponder a las presas secundarias, ya que la alimentación de los roedores (omnívoros, e.g., Abrothrix spp.) incluye artrópodos (Delibes et al. 2003).

TABLA 1. Dieta del quique (Galictis cuja) en un remanente de bosque valdiviano fragmentado del sur de Chile. En la última columna se señalan entre paréntesis las frecuencias absolutas de ocurrencia.

TABLE 1. Diet of the lesser grison (Galictis cuja) in a fragmented Valdivian remnant forest of southern Chile. In the last column are shown into parentheses the absolute frequencies of occurrence.

\begin{tabular}{|c|c|c|c|c|c|}
\hline Presas & $\begin{array}{c}\text { Frecuencia } \\
\text { ocurrencia }(\mathrm{F})\end{array}$ & $\begin{array}{c}\text { Frecuencia } \\
\text { ocurrencia }(F)\end{array}$ & $\begin{array}{c}\text { Frecuencia } \\
\text { ocurrencia } \\
\text { (F) }\end{array}$ & $\mathrm{F}(\%)$ & $\begin{array}{c}\text { Frecuencia } \\
\text { estandarizada }(\%)\end{array}$ \\
\hline & Otoño $(\mathrm{n}=15)$ & $\begin{array}{c}\text { Invierno } \\
(\mathrm{n}=30)\end{array}$ & Total $(n=45)$ & & \\
\hline \multicolumn{6}{|l|}{ Roedores } \\
\hline Abrothrix olivaceus & 9 & 15 & 24 & & \\
\hline Abrothrix longipilis & 4 & 9 & 13 & & \\
\hline Loxodontomys micropus & 4 & 10 & 14 & & \\
\hline Geoxus valdivianus & 0 & 4 & 4 & & \\
\hline Oligoryzomys longicaudatus & 1 & 3 & 4 & & \\
\hline Abrothrix spp. & 1 & 3 & 4 & & \\
\hline Subtotal roedores & 15 & 30 & 45 & 100 & $82(63)$ \\
\hline \multicolumn{6}{|l|}{ Insectos } \\
\hline Coleoptera & 2 & 3 & 5 & & \\
\hline Cratomelus armatus & 2 & 1 & 3 & & \\
\hline Subtotal insectos & 5 & 4 & 8 & 18 & $14(8)$ \\
\hline \multicolumn{6}{|l|}{ Aves } \\
\hline Especies no determinadas & 0 & 1 & 1 & & \\
\hline Subtotal aves & 0 & 1 & 1 & 2,2 & $2(1)$ \\
\hline \multicolumn{6}{|l|}{ Crustáceos } \\
\hline Aegla spp. & 0 & 1 & 1 & & \\
\hline Subtotal crustáceos & 0 & 1 & 1 & 2,2 & $2(1)$ \\
\hline Total frecuencias & 19 & 36 & 55 & & \\
\hline Total fecas & 15 & 30 & 45 & & \\
\hline
\end{tabular}


Llama la atención la presencia de restos de un crustáceo nativo (pancora) del género Aegla Leach 1820 en las fecas analizadas, presa que es común en la dieta del visón americano introducido, Neovison vison Schreber 1777 (Medina 1997). Sin embargo, en el área de estudio, donde existe un canal de irrigación que se llena de agua en invierno, hasta ahora no se han fotografiado visones con trampas-cámaras $\mathrm{y} / \mathrm{o}$ registrado sus huellas en estaciones de atracción olfativas. Al respecto, Delibes et al. (2003) han planteado la hipótesis que en hábitats donde quiques y visones americanos co-ocurren (i.e., viven en sintopía) éstos se segregarían en sus dietas implicando principalmente el consumo de presas terrestres en quiques y de presas acuáticas en visones. A pesar de lo anteriormente señalado, en el sur de Brasil se encontró la presencia de parásitos renales (Dioctophyma renale) (Barros et al. 1990, Pedrassani 2009) en quiques, los cuales son transmitidos por moluscos y crustáceos de agua dulce, presumiéndose con esto también el consumo de presas acuáticas por parte de este mustélido, lo que se contrapone con los escasos registros de este ítem en su dieta.

En el contenido estomacal e intestinal del animal atropellado durante la época estival se encontraron restos por molares de los roedores A. olivaceus, L. micropus, plumas del ave rapaz Athene cunicularia (pequén) y restos de coleópteros. En verano la oferta de roedores disminuye dramáticamente (cf. Murúa \& González 1986) por lo que el pequén, que se caracteriza por utilizar madrigueras o cuevas subterráneas de otros animales (Muñoz-Pedreros et al. 2004), sería una potencial presa alternativa dados los hábitos de caza del quique.

\section{AGRADECIMIENTOS}

La primera autora (SS) agradece a la Vicerrectoría de Investigación y Postgrado de la Universidad de Los Lagos por el financiamiento parcial de esta investigación la cual forma parte de su tesis de Magíster en Ciencias. También se agradece a los Sres. Gedike y Puschel por permitirnos el ingreso a sus predios. Además, se agradece al Dr. Alberto Gantz por su siempre buena disposición para la colaboración en el trabajo de campo y al Sr. Juan Cancino por ayudarnos con la edición final de las fotografías. Se agradece muy especialmente a los revisores Sonia Zapata y Fernando Alfaro por su contribución y valiosos aportes en la revisión crítica de este manuscrito. El segundo autor (JRR) agradece a Mateo Rau C. por la edición del texto y a Carlos Zuleta y Angel Spotorno por hacernos llegar bibliografía.
Finalmente, agradecemos a Agustín Iriarte por facilitarnos sus trampas-cámaras.

\section{BIBLIOGRAFIA}

Barros, DM., Lorini, ML. \& Persson, VG. 1990. Dioctophymosis in the little grison (Galictis cuja). Journal of Wildlife Diseases 26:538-539.

Delibes, M., Travaini A., Zapata, S.C. \& Palomares, F. 2003. Alien mammals and the trophic position of the lesser grison (Galictis cuja) in Argentinian Patagonia. Canadian Journal of Zoology 81: 157-162.

Dietrich, U. 1984. Beitrag zum status des Europäischen feldhasen (Lepus europaeus Pallas 1778) im südlichen Chile. Zeitschrift für Jagdwissenschaft 30: 256-259.

Diuk-Wasser, M.A. \& Cassini. M.H. 1998. A study of the diet of minor grisons and a preliminary analysis of their role in the control of rabbits in Patagonia. Studies on Neotropical Fauna and Environment 33: 3-6.

Ebensperger, L.A., Mella, J. E. \& Simonetti, J.A. 1991. Trophic relationships among Galictis cuja and Tyto alba in central Chile. Journal of Mammalogy 72: 820-823.

Kraus, C. \& RöDEL, H.G. 2004. Where have all the cavies gone? Causes and consequences of predation by the minor grison on a wild cavy population. Oikos 105: 489-500.

Maehr, D.S. \& Brady, J.R. 1986. Food habits of bobcats in Florida. Journal of Mammalogy 67: 133-138.

Medina, G. 1997. A comparison of the diet and distribution of southern river otter (Lutra provocax) and mink (Mustela vison) in southern Chile. Journal of Zoology (London) 242: 291-297.

Muñoz-Pedreros, A., Rau, J. \& Yañez, J. (eds.) 2004. Aves rapaces de Chile. CEA Ediciones. Valdivia, Chile.

Murua, R. \& Gonzalez, L.A. 1986. Regulation of numbers in two Neotropical rodent species in southern Chile. Revista Chilena de Historia Natural 59: 193-200.

Pedrassani, D. 2009. Aspectos morfológicos, imunológicos e epidemiológicos do Dioctophyme renale em Cães no Distrito de São Cristóvão, Três Barras, Santa Catarina. Tesis de Grado. Título de Doutor em Medicina Veterinária. Universidade Estadual Paulista "Julio de Mesquita Filho" Faculdade de Ciências Agrárias e Veterinárias Câmpus de Jaboticabal. Sao Paulo, Brasil.

Reglamento de la Ley de Caza Decreto Supremo No 05. 1998. Título II de la caza, captura, vedas y otras disposiciones relacionadas. $46 \mathrm{pp}$.

ReISE, D. 1973. Clave para la determinación de los cráneos de marsupiales y roedores chilenos. Gayana: Zoología 27: 320.

Yensen, E. \& Tarifa, T. 2003. Galictis cuja. Mammalian Species 728: $1-8$.

Zapata, S.C., Travaini, A, Delibes, M. \& Martinez-Peck, R. 2005. Annual food habits of the lesser grison (Galictis cuja) on the southern limits of its range. Mammalia 69: 85-88.

Recibido: 18.01 .12

Aceptado: 27.04.12 